\title{
An Unusual Case of a Pediatric Nasal Septal Abscess with Life- Threatening Complications in COVID-19 Pandemic
}

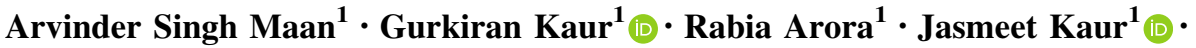 \\ Kamana Jyotsna Devi ${ }^{1}$ (D) Manjit Singh ${ }^{1}$
}

Received: 13 September 2020/ Accepted: 3 November 2020/Published online: 11 November 2020

(C) Association of Otolaryngologists of India 2020

\begin{abstract}
In this modern era, when access to healthcare services is improved, and awareness among the general population is enhanced, the presentation of mere septal abscess of the nose as a fatal complication is less common. Due to various lockdown restrictions in the COVID-19 scenario and fear to contract an infection, patients are presenting late to the health care setting for proper management. We treated an 11-year old child of complicated nasal septal abscess who responded well to aspiration of pus and medical treatment. Our patient is a rare case report who progressed from vestibulitis to septal abscess and further leading to sinusitis, orbital, and intracranial complications.
\end{abstract}

Keywords Nasal septal abscess - COVID-19 .

Vestibulitis · Complications

Gurkiran Kaur

gurkiranmarwah23@gmail.com

Arvinder Singh Maan

maanarvinder@yahoo.com

Rabia Arora

rabia3001@gmail.com

Jasmeet Kaur

jazzrataul@gmail.com

Kamana Jyotsna Devi

jyotsnakamana@gmail.com

Manjit Singh

drmanjitsingh62@yahoo.com

1 Department of Otorhinolaryngology and Head and Neck Department, Ram Lal Eye and ENT Hospital, Government Medical College, Majitha Road, Amritsar, Punjab 143001, India

\section{Introduction}

In the post-antibiotic era, nasal septal abscess (NSA) is a rare pathology causing complications. Moreover, in the growing child, wholly or partially destroyed septal cartilage as a complication of abscess formation can hinder the normal midface development [1]. It is the immune-compromised patients in which rapid intracranial extension of NSA usually occurs [2]. However, we hereby report a pediatric immunocompetent case of NSA as sequelae of vestibulitis leading to sinusitis, and further progressing to facial cellulitis, pre-septal cellulitis, and meningitis.

\section{Case Report}

An 11 year old male child was admitted to the otorhinolaryngology and head and neck department of our institute with the chief complaint of high-grade fever, swelling over the nose, and eyelids for 4 days (Fig. 1). The patient also complained of pain in and around the nose and both eyes. There was no associated history of nasal discharge, nasal obstruction, mouth breathing, cough, difficulty in breathing, or any decreased sense of smell. According to patient's father, there was an initial onset of nasal swelling for which oral medication was taken from some local practitioner only due to COVID-19 restrictions, but no relief of symptoms was obtained; instead, swelling worsened further and involved both eyes. There was no history of nasal trauma, dental infection, and any significant past medical, surgical, and family history.

When the child visited the hospital, he was alert, conscious, and oriented to time, place, and person with no difficulty in breathing. The temperature on arrival was $102{ }^{\circ} \mathrm{F}$ orally, oxygen saturation of $99 \%$, heart rate of 100 


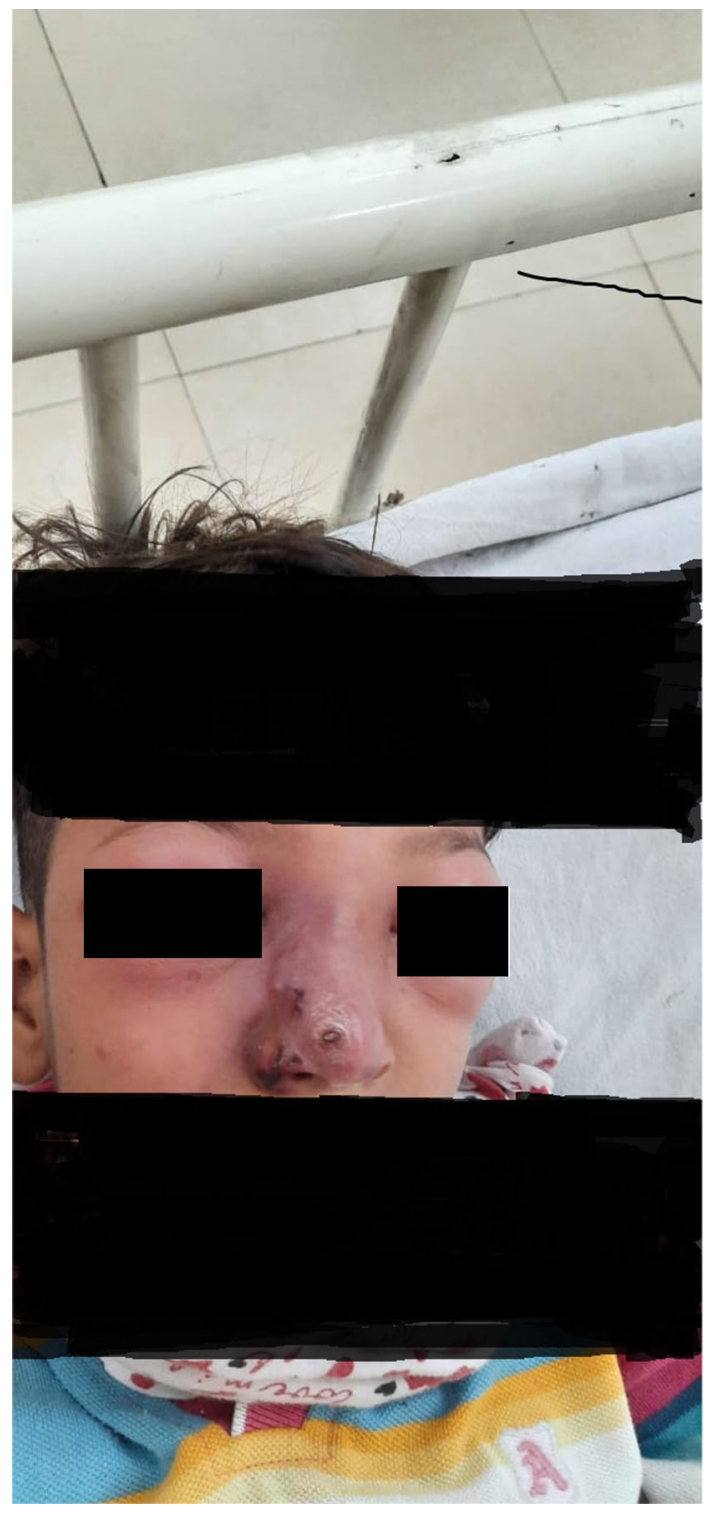

Fig. 1 The illustration of child on presentation showing swollen and erythematous bilateral eyes and nose. The blackish crusts are visualized on right alar and tip region

beats/min, respiratory rate of 20 breaths/min, and blood pressure of 104/90 mm Hg. No external deformity of the nose was appreciated on local examination, but the swelling, erythema, and blackish crusts more on the right alar region and tip was observed. The nose was tender on palpation, and slight boggy swelling was noted on the anterior nasal septum on right side. Ophthalmology opinion revealed swelling and erythema of eyelids and periorbital region, without any chemosis or conjunctival hemorrhage and fundus glow was present bilaterally but at that time, the child was uncooperative for bedside visual acuity and extraocular movements examination due to pain. Later all cranial nerve examinations was found to be normal.
Due to this ongoing COVID-19 pandemic and patient presenting with fever, an immediate nasopharyngeal and oropharyngeal swab was also sent, which turned out to be negative. All routine blood investigations were done. The total leucocyte count was raised to 13,660 cells $/ \mathrm{mm}^{3}$ with a neutrophil differential count of $84 \%$. The patient was sent for a contrast-enhanced computed tomography (CECT) Nose and paranasal sinuses (PNS) which was suggestive of a well defined oval hypodense collection with an average HU value of 25 , measuring $24 \times 21 \times 15.5 \mathrm{~mm}$ in size with peripheral enhancement involving the anterior part of the septum (Fig. 2) along with marked fat stranding in subcutaneous tissue overlying the nose, nasion, and bilateral orbits. Moreover, mucosal thickening was seen in all the sinuses.

The diagnostic nasal endoscopy revealed swelling involving the anterior nasal septum on the right side only. The needle aspiration of about $2.5 \mathrm{ml}$ pus was done which led to the resolution of septal swelling (Fig. 3) and was sent for culture sensitivity, which came out to be sterile because of ongoing antibiotic treatment. The child was started initially on intravenous ceftriaxone, metronidazole, paracetamol infusion, mupirocin local application, and oral ibuprofen.

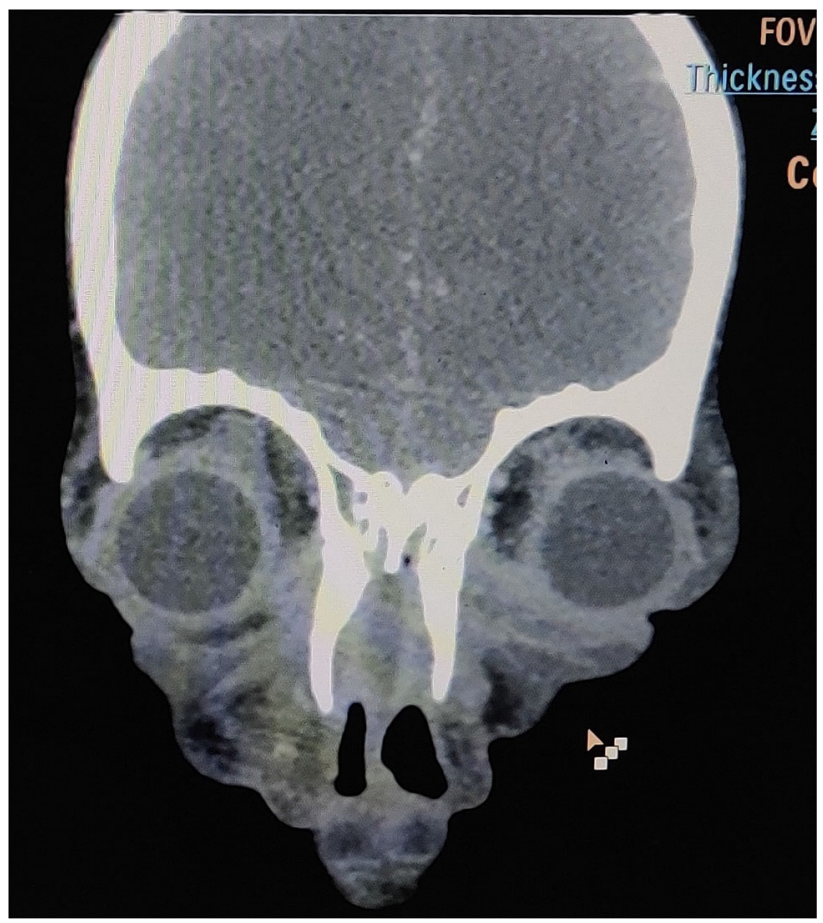

Fig. 2 The coronal section of CECT Nose and PNS showing hypodense lesion of septum with peripheral rim enhancement and typical fat stranding seen in right superior orbit region. (characteristic of pre-septal cellulitis) 


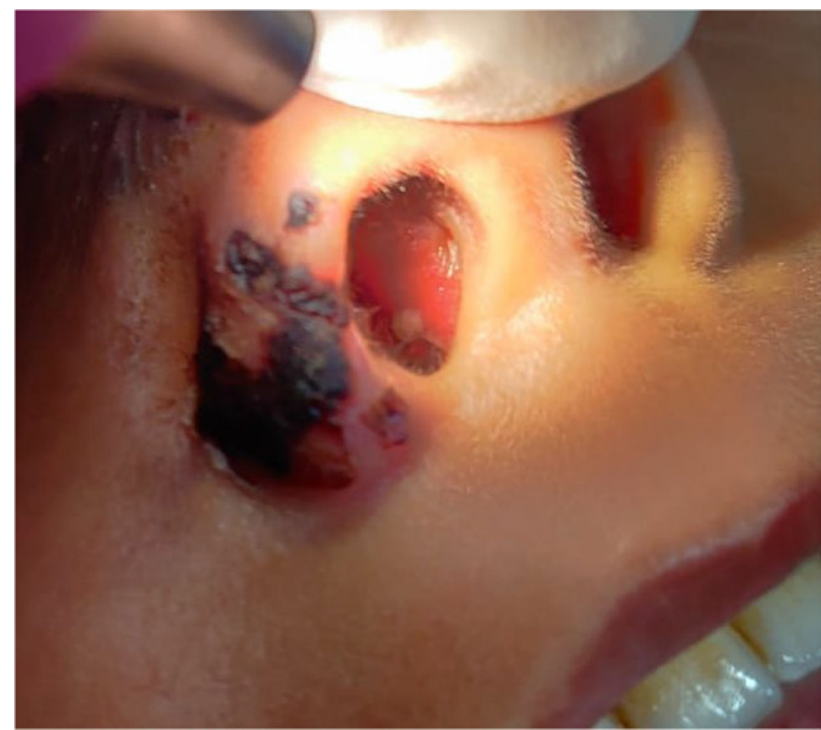

Fig. 3 The illustration of child post needle aspiration of pus showing resolution of boggy swelling on right side

After $4 \mathrm{~h}$ of admission, the child started developing features of meningismus such as headache, neck pain, projectile vomiting, photophobia, with already existing high-grade fever with no sign of altered sensorium. Then urgent MRI brain was done to rule out any intracranial complication. On a contrast MRI brain study, subtle meningeal enhancement was seen with meningitis suspicion (Fig. 4). There was no thrombus or filling defect in the cavernous sinus which ruled out its involvement. Overall

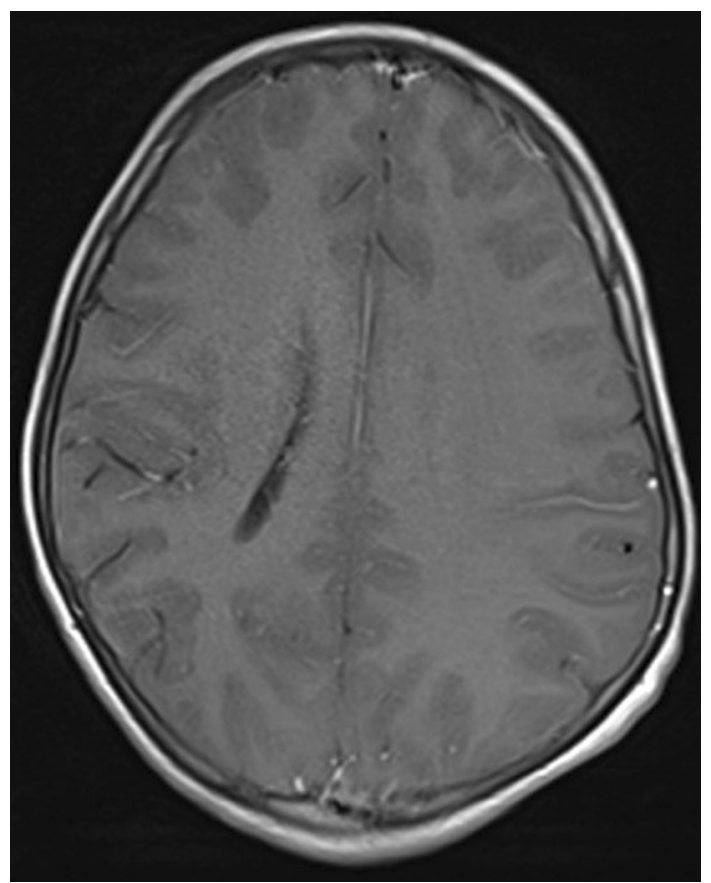

Fig. 4 The post contrast MRI image showing enhancement in sulcogyral region on left side of brain findings were suggestive of NSA involving the cartilaginous part of the septum as sequelae of vestibulitis of the nose and mild to moderate acute pansinusitis with bilateral pre-septal cellulitis with suspicion of meningitis.

The pediatrics consultation was taken and was advised to shift to a pediatric intensive care unit (ICU) under the joint care of the otorhinolaryngology and pediatrics department for medical management. The empirical therapy of intravenous vancomycin ( $40 \mathrm{mg} / \mathrm{kg} /$ day $)$, imipenem, mannitol, and fluconazole along with nasal oxy-xylometazoline drops was added. The child responded well to aspiration of pus and medical management and was discharged after full recovery in 14 days on oral antibiotic therapy for a week.

\section{Discussion}

NSA is defined as a collection of pus in the subperichondrial and subperiosteal plane of the septum. NSA occurs mostly in children with male predominance. The reason for this predominance could be explained that the mucoperichondrium and mucoperiosteum loosely adhere to the septum in children, and males are more involved in violent activities [3].

In about three-fourths of cases, the etiological agent is identified to be trauma followed by causes secondary to sinusitis, vestibulitis, dental pathologies with rare reporting of spontaneous cases [4]. Mostly NSA patients present with nasal obstruction with associated symptoms of pain, fever, malaise, headache, purulent nasal discharge, and delayed presentations can be cosmetic deformity of the nose such as saddle nose or any intracranial complication. According to the Korean case series, nasal obstruction is one of the initial, and nasal pain is the most common symptom reported in NSA [3]. The characteristic appearance of NSA on anterior rhinoscopy and diagnostic nasal endoscopy is septal swelling (bilateral $>$ unilateral). The usual bilateral involvement can be credited to septal cartilage resorption as a consequence of ischemia and necrosis [1].

The most common cause of NSA is aerobic bacteria to be specific; Staphylococcus aureus accounts for the majority of cases in adults and Haemophilus influenza in the pediatric population [4]. In the literature, the complications of NSA are categorized into: local, systemic, orbital, and cranial [3]. In the present case, local complications of sinusitis, vestibulitis, facial cellulitis, orbital complication of pre-septal cellulitis, and cranial complications of meningitis were observed.

These types of cases can progress from NSA to necrotizing fascitis stage and cavernous sinus thrombosis, as reported by Swaminath et al., if the further delay in management occurs. The intracranial extension can be via the 
perineural sheath, lymphatics, and hematogenous route [5]. Even retrograde infection from sinusitis and orbital cellulitis to NSA has been evident in the literature. The infection can travel to and fro due to the valve-less nature of the facial venous system [6].

An appropriate and timely radiological investigation is required in NSA cases. CECT nose and PNS's role is not only for diagnosis but also for ruling out any complication or the suspicion of rhinosinusitis, granulomatous diseases, sarcoma, or lymphoma in spontaneous cases [7]. It is to be noted that NSA has a characteristic appearance on CT examination as a cystic collection of fluid with thin rim enhancement involving the nasal septum [8]. The typical fat stranding is seen in facial cellulitis, but gas on radiography points towards the necrotizing fascitis stage [5]. MRI brain with contrast is undeniably beneficial in the preliminary investigations of suspicious intracranial extension and can avoid lethal complications.

Early diagnosis and immediate surgical intervention are necessary along-with parenteral antibiotic treatment. Simple needle aspiration has a role only for the diagnosis of an abscess and reducing pressure symptoms but is not sufficient for NSA management, and proper incision and drainage are required to prevent recollection. Apart from Killian's incision, horizontal longitudinal or L shaped incision are other options [2]. The drainage of abscess bilaterally is required only if septal cartilage is intact, and pus is collected on both sides, but usually, partial destruction of cartilage is present to allow common cavity and drainage by single incision [9]. Bilateral nasal packing is required postoperatively for a minimum $48 \mathrm{~h}$ with the controversial role of Penrose drain. As few studies like Canty and Berkowitz [10] and Kwak et al. [3] reported no benefit of Penrose drain concerning to re-accumulation of fluid. But in our case, only the anterior nasal septum was involved on one side without septal cartilage destruction and simple aspiration gave good results without recurrence.

\section{Conclusion}

NSA is itself an infrequent pathology in today's scenario and complicated NSA cases are rare. It should be taken into consideration that NSA can be atraumatic with no history of nasal obstruction, but a lack of prompt diagnosis can land up the patient in life-threatening complications. Due to the COVID-19 pandemic, people are apprehensive of reporting to a hospital because of the social stigma of contracting an infection, and further restriction of movement is making the situation worse.

\section{Compliance with Ethical Standards}

Conflict of interest The study has not received funding from any organization or institution and does not involve any potential conflict of interest (financial and non-financial).

Ethical Approval Procedure performed in the study was in accordance with the ethical standards of the institution and with the 1964 Helsinki declaration and its later amendments.

Informed Consent Informed consent was obtained from patient's guardian in the study.

\section{References}

1. Alshaikh N, Lo S (2011) Nasal septal abscess in children: from diagnosis to management and prevention. Int J Pediatr Otorhinolaryngol 75(6):737-744. https://doi.org/10.1016/j.ijporl. 2011.03.010

2. Adnane C, Adouly T, Taali L et al (2015) Unusual Spontaneous Nasal Septal Abscess. J Case Rep Stud 3:302. https://doi. org/10.15744/2348-9820.3.302

3. Kwak KH, Lee J, Lim EJ, Park JH, Kim SH, Kim TH. Nasal Septal Abscess: Clinical Analysis of 6 Cases. Journal of Clinical Otolaryngology. 2015; 26:213-218. https://doi.org/10.35420/ jcohns.2015.26.2.213

4. Hyo Y, Fukushima H, Harada T, Hara H (2018) Nasal septal abscess caused by anaerobic bacteria of oral flora. Auris Nasus Larynx 46:147-150. https://doi.org/10.1016/j.anl.2018.05.014

5. Swaminath D, Narayanan R, Orellana-Barrios MA, Temple B (2014) Necrotizing fasciitis of the nose complicated with cavernous sinus thrombosis. Case Rep Infect Dis. https://doi.org/10.1155/2014/914042

6. Fatima SN, Sarwar F, Khan (2018) Nasal septal abscess as a sequela of orbital cellulitis: an uncommon presentation. SAGE Open Med Case Rep 6:2050313X18778726. https://doi.org/10. 1177/2050313X18778726

7. Nwosu JN, Nnadede PC (2015) Nasalseptal hematoma/abscess: management and outcome in a tertiary hospital of a developing country. Patient Preference Adher 9:1017-1021. https://doi.org/ 10.2147/PPA.S85184

8. Debnam JM, Gillenwater AM, Ginsberg LE (2007) Nasal septal abscess in patients with immunosuppression. Am $\mathrm{J}$ Neuroradiol 28:1878-1879. https://doi.org/10.3174/ajnr.a0708

9. Shapiro RS (1978) Nasal septal abscess. CMA 119(11) 1321-1323

10. Canty PA, Berkowitz RG (1996) Hematoma and abscess of the nasal septum in children. Arch Otolaryngol Head Neck Surg 122(12):1373-1376. https://doi.org/10.1001/archotol.1996. 01890240079017

Publisher's Note Springer Nature remains neutral with regard to jurisdictional claims in published maps and institutional affiliations. 